\title{
KCl-Dependent Release of Mitochondrial Membrane-Bound Arginase Appears to Be a Novel Variant of Arginase-II
}

\author{
Mishra Suman and Mishra Rajnikant \\ Biochemistry and Molecular Biology Laboratory, Department of Zoology, Banaras Hindu University, Varanasi 221005, India \\ Correspondence should be addressed to Mishra Rajnikant; rmishraa@bhu.ac.in
}

Received 7 December 2015; Revised 7 April 2016; Accepted 18 April 2016

Academic Editor: Ariel M. Silber

Copyright ( 2016 M. Suman and M. Rajnikant. This is an open access article distributed under the Creative Commons Attribution License, which permits unrestricted use, distribution, and reproduction in any medium, provided the original work is properly cited.

\begin{abstract}
Arginase regulates arginine metabolism, ornithine-urea cycle, and immunological surveillance. Arginase-I is predominant in cytosol, and arginase-II is localised in the mitochondria. A mitochondrial membrane-bound arginase has also been proposed to be adsorbed with outer membrane of mitochondria which gets released by $150 \mathrm{mM}$ potassium chloride (KCl). It is presumed that inclusion of $150 \mathrm{mM} \mathrm{KCl}$ in the homogenization medium would not only facilitate release of arginase bound with outer membrane of mitochondria but also affect functional anatomy of mitochondria, mitochondrial enzymes, and proteins. Therefore, it has been intended to characterize $\mathrm{KCl}$-dependent release of mitochondrial membrane-bound arginase from liver of mice. Results provide advancement in the area of arginase biology and suggest that fraction of mitochondrial membrane-bound arginase contains mitochondrial arginase-II and a variant of arginase-II.
\end{abstract}

\section{Introduction}

Arginase [E.C 3.5.3.1] catalyzes synthesis of ornithine and urea from arginine [1]. It regulates level of L-arginine [2] and production of nitrous oxide (NO) [3]. It has also been important for immunological surveillance $[4,5]$. It gets upregulated in vascular abnormalities [6,7], diabetes [8], hepatocellular carcinoma [9], cardiovascular diseases [10], and neuroinflammation [11]. Other roles of arginase have also been reviewed [12], showing impact of arginase on biological significance of arginine metabolism associated pathological conditions. Two major isoforms of arginase, cytosolic arginase-I and mitochondrial arginase-II, have been described in different organisms [13-16] as having distinct physicochemical, molecular, and immunological properties. Several other isoforms have also been reported on the basis of difference in net-charge, immunological properties, and subcellular localization. Three isoforms of arginase have been observed in rat liver on the basis of elution pattern of CMcellulose chromatography [17] and electrophoresis pattern [18]. Four isoforms of arginase in frog and lizard on the basis of DEAE-cellulose chromatography [19] and five isoforms of arginase in rat on the basis of immunological properties [20] have also been proposed. One of the forms of arginase has also been proposed to bind with external side of inner mitochondrial membrane of chicken kidney [21]. However, cytosolic arginase-I has also been thought to be associated at the surface of subcellular organelles [22]. The activity of arginase has also been observed [23-25] in the fractions extracted with $150 \mathrm{mM}$ potassium chloride $(\mathrm{KCl})$ or butanol [26]. The use of $\mathrm{KCl}$ in homogenizing medium for isolation of mitochondria has resulted in increased arginase activity in cytosolic fraction and decreased arginase activity in mitochondrial fraction [25]. However, the high salt concentration [27, 28] may affect mitochondrial membrane. It is presumed that $150 \mathrm{mM}$ $\mathrm{KCl}$ may not only facilitate release of arginase bound with membrane of mitochondria but also affect integrity of mitochondria and other cell organelles. It is likely that arginaseII gets released from mitochondria due to alterations in permeability or damage of mitochondrial membrane under nonoptimal $\mathrm{KCl}$ concentration. The posttranslational modification of arginase-II may also alter targeting or translocation to the mitochondria that may remain adhered either to outer or to inner membrane (Figure 8) of mitochondria. The activity of mitochondrial membrane-bound arginase has been indicated but biochemical and molecular characterization 
of the mitochondrial membrane-bound arginase is limiting. Therefore, it has been intended to evaluate $\mathrm{KCl}$-dependent activity of mitochondrial membrane-bound arginase from mitochondria isolated from mice liver. The results suggest that $\mathrm{KCl}$-dependent release of mitochondrial membranebound arginase appears to be a variant of arginase-II.

\section{Materials and Methods}

2.1. Animals and Materials. The adult ( $12 \pm 2$ weeks) mice (Mus musculus) of AKR strain were maintained with standard mice feed and drinking water at $25 \pm 2^{\circ} \mathrm{C}$ in animal house facility of the department as per guidelines of the Institutional Animal Ethical Committee. Animals were sacrificed to obtain liver. All analytical grade chemicals were used and purchased from local suppliers. Anti-arginase-I (sc-20150), antiarginase-II (sc-20151), COX II (sc-23983), and anti-GAPDH (sc-25778) were purchased from Santa-Cruz Biotechnology Inc. (USA) and anti-cytochrome C (C5723) was purchased from Sigma-Aldrich (USA). They are well characterized and widely used antibodies in the literature.

\subsection{Effect of $\mathrm{KCl}$ on Mitochondrial Membrane Associated} Arginase. The experimental plan of isolation of cytosol, mitochondria, and mitochondrial membrane-bound arginase was summarized in Figure 1. In brief, $20 \%$ homogenate of liver tissue was prepared in homogenizing buffer containing $30 \mathrm{mM}$ Tris $\mathrm{HCl}$ (pH 7.2), $1 \mathrm{mM}$ EDTA, $250 \mathrm{mM}$ sucrose, $50 \mathrm{mM}$ mannitol, and protease inhibitor cocktail (Sigma-Aldrich) using a Potter-Elvehjem type glass homogenizer with a motor-driven teflon pestle. Homogenate was centrifuged at $600 \times \mathrm{g}$ for $10 \mathrm{~min}$ and pellet was discarded. The supernatant was further centrifuged at $12,000 \times \mathrm{g}$ for $15 \mathrm{~min}$ for isolation of mitochondria. The supernatant was considered as cytosolic fraction (C) and pellet was washed twice with homogenizing buffer (without $\mathrm{KCl}$ ) and centrifuged at 12,000 $\times \mathrm{g}$ for $15 \mathrm{~min}$. Both supernatants (W1 and W2) were analysed to confirm the release of mitochondrial matrix arginase or contamination of cytosolic arginase in mitochondrial pellet. The pellet obtained after second wash (W2) was considered as total mitochondrial pellet (Tmt). For the isolation of mitochondrial membrane-bound fraction, the washed mitochondrial pellet (Tmt) was suspended with $\mathrm{KCl}$-containing homogenizing buffer $(100 \mathrm{mM} \mathrm{KCl})$ and centrifuged at $12,000 \times \mathrm{g}$ for $15 \mathrm{~min}$. The supernatant was considered as the mitochondrial membrane-bound fraction (MtMb) and the pellet as the washed mitochondrial matrix fraction (Mtm).

The first set of experiment was like earlier reports to evaluate effects of $\mathrm{KCl}$ when used in homogenization medium before isolation of mitochondria. In first set, different concentrations of $\mathrm{KCl}(0,25,50,100,150$, and $200 \mathrm{mM})$ were used in homogenizing medium, and mitochondrial and cytosolic fractions (C) were isolated. The second set of experiment was planned to confirm that the activity of arginase was due to $\mathrm{KCl}$ treatment on the mitochondrial membrane, not contributed by membrane of any other subcellular organelle. In second set, mitochondria were isolated first without $\mathrm{KCl}$ in homogenizing medium. Washed mitochondrial pellet (total mitochondria, Tmt) was suspended in different concentrations of $\mathrm{KCl}(0,25,50,100,150$, and $200 \mathrm{mM})$ in homogenizing medium. Then, membrane-bound (MtMb) and mitochondrial fractions (Mtm) were separated. These fractions were used for arginase assay and western blot analysis. The $100 \mathrm{mM} \mathrm{KCl}$ was used in validation of subcellular fractionation.

The extraction of cytosolic, mitochondrial, and membrane-bound fraction was evaluated by mitochondrial marker enzyme assay. The activity of succinate dehydrogenase (SDH) was measured by the spectrophotometric method of Ells [29] in $1 \mathrm{~mL}$ reaction mixture containing $67.5 \mathrm{mM}$ potassium phosphate buffer $(\mathrm{pH} 8.5), 16.6 \mathrm{mM}$ succinate, $2 \mathrm{mM}$ nicotinamide adenine dinucleotide $\left(\mathrm{NAD}^{+}\right)$, $2 \mathrm{mM}$ phenazine methosulfate (PMS), and 2,6-dichlorophenolindophenol (DCPIP). The continuous assay started after addition of enzyme source and absorbance was recorded at $600 \mathrm{~nm}$ up to $3 \mathrm{~min}$. Difference in absorbance was used for the calculation of activity. One unit of SDH activity was expressed as the amount that utilized $1 \mu \mathrm{mole}$ of $\mathrm{NAD}^{+}$ per min at $30^{\circ} \mathrm{C}$. Protein was estimated by the method of Bradford [30] taking bovine serum albumin as standard.

Arginase assay was performed according to the method of Brown Jr. and Cohen [31]. The reaction mixture consisting of $25 \mathrm{mM}$ sodium glycinate buffer ( $\mathrm{pH} 9.5$ ), $2.5 \mathrm{mM} \mathrm{MnCl}_{2}$, $25 \mathrm{mM}$ L-arginine, and suitably diluted enzyme extract, with total volume of $2 \mathrm{~mL}$, was incubated for 15 minutes at $30^{\circ} \mathrm{C}$. The reaction was terminated by adding $10 \%$ perchloric acid, and protein was removed by centrifugation. Urea was determined in the supernatant. One unit of arginase activity was defined as the amount of enzyme, which produced one $\mu$ mole of urea per hour at $30^{\circ} \mathrm{C}$.

2.3. Analysis of Expression Pattern of Arginase-I and ArginaseII by Western Blot and RT-PCR. Proteins of cytosolic, mitochondrial, and mitochondrial membrane-bound fractions were resolved on $15 \%$ denaturing gel. Resolved polypeptides were transferred on polyvinylidene difluoride (PVDF) membrane. Membrane was blocked with 5\% nonfat milk in $1 \mathrm{x}$ TBS-T for one hour after the transfer of protein. After blocking, the transferred membrane was incubated with anti-arginase-I and anti-arginase-II at 1:500 dilutions in $2 \%$ nonfat milk in $1 \mathrm{x}$ TBS-T for overnight at $4^{\circ} \mathrm{C}$ for both. The membrane was washed four times for fifteen minutes each after primary antibody incubation. Membrane was incubated with secondary antibody (goat anti-rabbit IgG antibody, Genei) at 1:5000 dilutions in 2\% nonfat milk in 1x TBS-T for 2 hours for all four antibodies used. Anti-cytochrome $\mathrm{C}$ and anti-GAPDH were used as positive controls at 1:1000 dilutions, respectively. For validation of subcellular fractionation, cytosolic marker GAPDH at 1:1000 and mitochondrial marker COX II (sc-23983) at 1:500 dilutions were used. Immunodetection was performed using the enhanced chemiluminescence (ECL) system. The signals of chemiluminescence were detected and analysed through LAS 500, Chemi-doc (GE Healthcare) imaging system.

Total RNA was extracted from liver using TRIzol (Invitrogen). The RNA was quantified by spectrophotometric analysis and the quality was checked on $1 \%$ agarose gel. Isolated RNA was reverse transcribed using Oligo-dT 


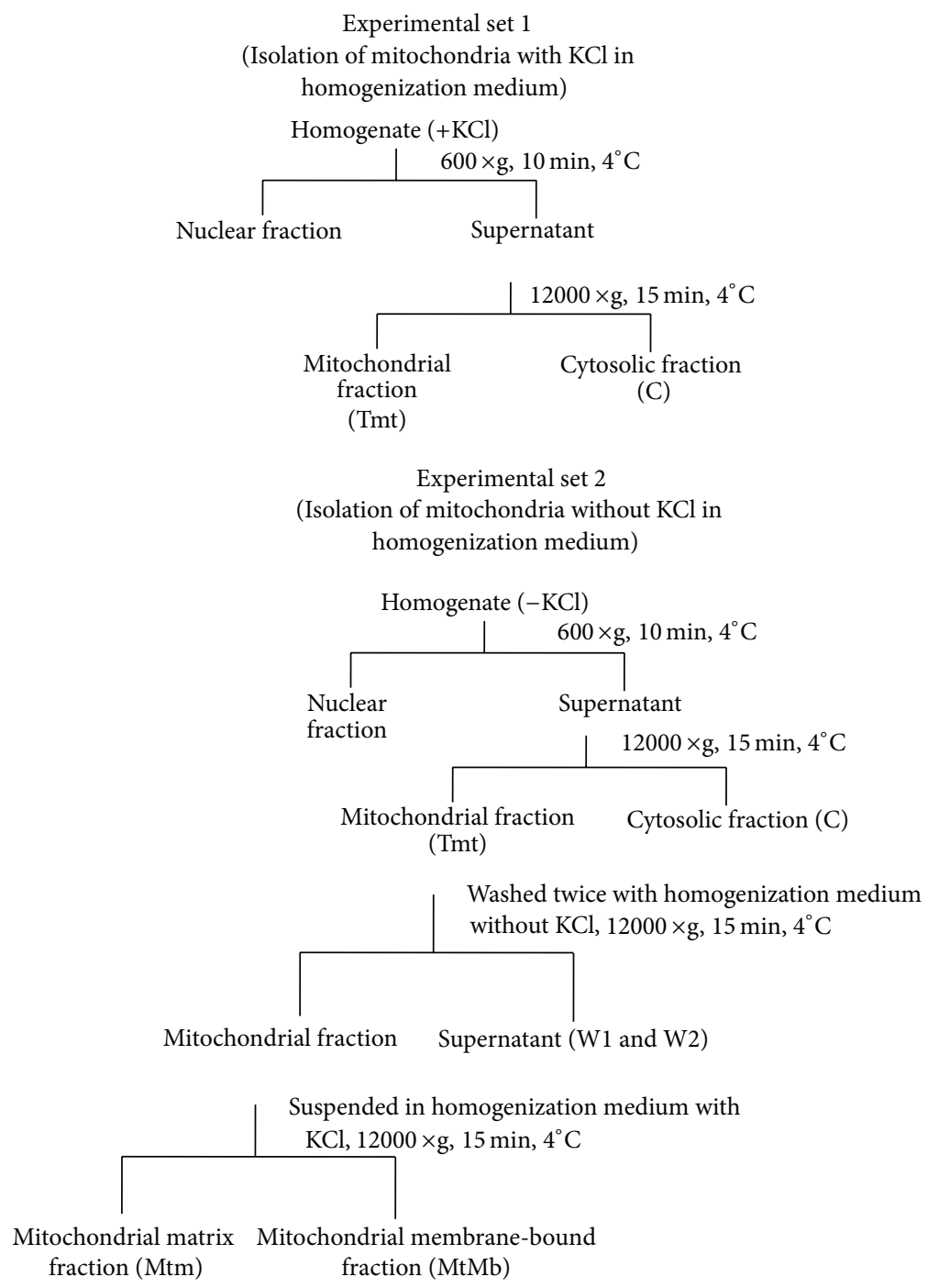

FIGURE 1: Flow chart of the procedure of isolation of cytosolic fraction, mitochondrial fraction, and mitochondrial outer membrane-bound fraction. The experimental set 1 mitochondria were isolated with $\mathrm{KCl}$ in homogenizing medium and experiment set 2 in which mitochondria were isolated first and then washed with different concentration of $\mathrm{KCl}$ in homogenizing buffer.

primer according to suggested protocol for first strand cDNA synthesis kit (Applied Biosystems). The RT-PCR was performed by using primers RARGIF $5^{\prime}$ GTCCAGAAGAATGGAAGAGTCAG3'; RARGIflR $5^{\prime}$ CGTGGATATAGGCTACC $3^{\prime}$; RARGIIF $5^{\prime}$ GCACTCACTCGAGGTCCTG3'; and RARGFIIR $5^{\prime}$ GACTCCTTCAAACTTACATG3'. The PCR products were analysed on $1 \%$ agarose gel.

2.4. In Silico Analysis of Arginase-I and Arginase-II. The putative mitochondrial targeting of arginase-I and arginaseII was also analysed in silico. The GenBank sequences for arginase-I (AAA98611.1) and arginase-II (AAC22548.1) were obtained from NCBI (http://www.ncbi.nlm.nih.gov/) and were studied for presumed subcellular targeting. The subcellular localization was predicted by MitoProt-II (http:// ihg.gsf.de/ihg/mitoprot.html) and iPSORT (http://ipsort .hgc.jp/predict.cgi) servers.
Gene and transcript analyses of arginase-I and arginase-II were done by UCSC genome browser (https://genome.ucsc .edu/) and NCBI Ensembl (http://www.ensembl.org/) databases. EST was searched in NCBI EST database (http:// www.ncbi.nlm.nih.gov/nucest/). Also, alternative splice variants were studied by ASSP (http://wangcomputing.com/ assp/). The physicochemical properties and posttranslational modification were predicted by ExPAsy proteomic tool (http://www.expasy.org/proteomics) also described in Table 1.

2.5. Electron Microscopy of Mitochondria. The mitochondrial pellets of control were isolated without $\mathrm{KCl}$ and treated (isolated with $100 \mathrm{mM} \mathrm{KCl}$, as they were found to have intermediate concentration) by the protocol of second experiment set. The mitochondrial pellets were fixed in $2.5 \%$ glutaraldehyde and $1 \%$ paraformaldehyde in phosphate buffer $(\mathrm{pH} 7.5)$ at $4^{\circ} \mathrm{C}$ 
TABLE 1: Comparative properties of Mus musculus arginase-I and arginase-II.

\begin{tabular}{|c|c|c|}
\hline Parameters & Arginase-I (AAA98611.1) & Arginase-II (AAC22548.1) \\
\hline Molecular weight & 34807.8 & 38878.3 \\
\hline $\mathrm{pI}$ & 6.52 & 6.10 \\
\hline Total number of negatively charged residues $(\mathrm{Asp}+\mathrm{Glu})$ & 39 & 40 \\
\hline Total number of positively charged residues (Arg + Lys) & 37 & 33 \\
\hline Aliphatic index & 90.77 & 97.46 \\
\hline $\begin{array}{l}\text { Grand average of hydropathicity (GRAVY) } \\
\text { (http://www.expasy.org/proteomics) }\end{array}$ & -0.187 & -0.109 \\
\hline $\begin{array}{l}\text { Mitochondrial import } \\
\text { (https://ihg.gsf.de/ihg/mitoprot.html) }\end{array}$ & No $(0.060)$ & Yes $(0.9771)$ \\
\hline $\begin{array}{l}\text { N-phosphorylation sites } \\
\text { (http://www.cbs.dtu.dk/services/NetPhos/) }\end{array}$ & $\begin{array}{c}20 \text { sites ( } 9 \text { serine residues, } 6 \\
\text { threonine residues, and } 5 \\
\text { tyrosine residues) }\end{array}$ & $\begin{array}{c}18 \text { sites (one site } S^{6}(0.974) \text { in } \\
\text { mitochondrial targeting } \\
\text { sequence) }\end{array}$ \\
\hline $\begin{array}{l}\mathrm{N} \text {-acetylation sites } \\
\text { (http://www.cbs.dtu.dk/services/NetAcet/) }\end{array}$ & $\begin{array}{c}2 \text { sites at } 2 \text { nd S (score } 0.501 \text { ) and } \\
\text { 3rd S (score 0.492) from } \\
\text { N-terminus }\end{array}$ & $\begin{array}{l}\text { No acetylation sites were found } \\
\text { from N-terminus }\end{array}$ \\
\hline $\begin{array}{l}\text { O-GlcNAcetylation sites } \\
\text { (http://www.cbs.dtu.dk/services/YinOYang/) }\end{array}$ & 9 sites & $\begin{array}{c}7 \text { sites (no sites were found in } \\
\text { mitochondrial targeting } \\
\text { sequence) }\end{array}$ \\
\hline $\begin{array}{l}\text { N-glycosylation sites } \\
\text { (http://www.cbs.dtu.dk/services/NetNGlyc/) }\end{array}$ & 5 sites & $\begin{array}{c}5 \text { sites (no sites in mitochondrial } \\
\text { targeting sequence) }\end{array}$ \\
\hline
\end{tabular}

overnight. The fixed mitochondrial pellets were dehydrated through grades of alcohol (30\% to absolute alcohol), and the preparation of block, sectioning, and staining for TEM analysis was executed at AIIMS, New Delhi.

2.6. Statistical Analysis. The values are expressed as the mean \pm SD. The data were analysed by one-way analysis of variance (ANOVA) followed by Tukey's test. The level of significance is taken at $P<0.05$.

\section{Results}

3.1. The Cytosolic, Mitochondrial, and Mitochondrial Membrane-Bound Fractions Show Presence of Suitable Biochemical Markers. The cytosolic, mitochondrial, and mitochondrial membrane-bound fractions were isolated by differential centrifugation method and validated by the spectrophotometric analysis of SDH (Figure 2(a)) and western blot analysis by GAPDH and COX II (Figure 2(b)). The spectrophotometric analysis did not detect activity in mitochondrial membrane-bound fraction but some activity was observed in cytosolic fraction, which may be due to disruption of mitochondria during handling (Figure 2(a)). The anti-GAPDH did not show any immunoreactive band in mitochondrial and mitochondrial membrane-bound fraction that could be considered as the mitochondrial fraction was free from the cytosolic contamination. The COX II detected band in mitochondrial matrix and mitochondrial membrane-bound fraction (Figure 2(b)).

3.2. Expression Pattern and Activities of Arginase Were Progressively Affected by $\mathrm{KCl}$. The arginase activity was detected in cytosolic fraction (C), supernatant of first mitochondrial wash (W1), supernatant of second mitochondrial wash (W2), mitochondrial matrix fraction (Mtm), total mitochondrial fraction (TMt), and mitochondrial membrane-bound fraction (MtMb). However, the maximum specific activity was observed in mitochondrial membrane-bound fraction (Figure 3(a)). Since arginase assay system could not differentiate the isoform of arginase, western blot analysis was performed in all fractions and probed with anti-arginase-I, antiarginase-II, GAPDH, and cytochrome C. The anti-arginase-I reactive band was observed (Figure $3(\mathrm{~b})$ ) in cytosolic fraction but no bands were seen in mitochondrial and mitochondrial membrane-bound fraction. However, single band was detected in both washed fractions with anti-arginase-I and anti-arginase-II. The anti-arginase-II showed two immunoreactive bands in the mitochondrial membrane-bound fraction but did not detect any immunoreactive band in cytosolic fraction. One of the two bands, matched with canonical arginase-II while having an additional immune-reactive band (marked by arrow) identified by arginase-II, was observed specifically in mitochondrial fraction (Figure 3(b)).

The activity of arginase (Figure 4(a)) was observed to be progressively higher in cytosolic fraction, whereas activity of arginase in mitochondrial fraction (Figure 4(c)) was lower in experiment set 1 . Similar pattern was found in western blot analysis by anti-arginase-I and anti-arginase-II in cytosol and mitochondrial fraction (Figures $4(\mathrm{~b})$ and $4(\mathrm{~d})$ ). The activity of arginase increased in mitochondrial membranebound fraction (Figure 5(c)) but decreased in mitochondrial fraction (Figure 5(a)) in experiment set 2. The decrease in activity of arginase in mitochondrial fraction was similar to the increase in membrane-bound fraction. However, in the western blot analysis, an additional band with antiarginase-II was observed which was also increasing with 


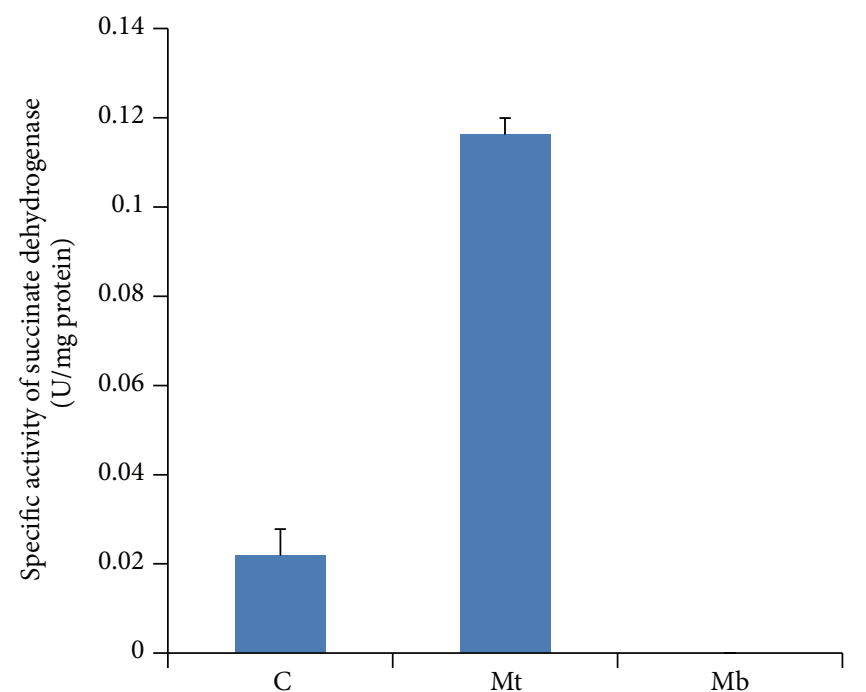

(a)

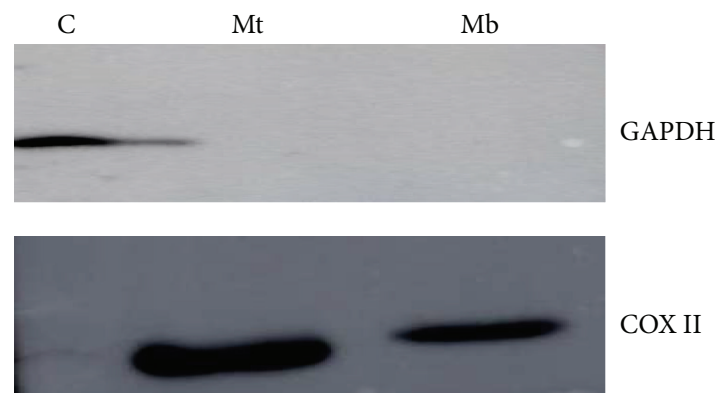

(b)

FIGURE 2: (a) Specific activity analysis of succinate dehydrogenase (SDH). (b) Western blot analysis of cytosolic GAPDH and mitochondrial COX II in $\mathrm{C}=$ cytosol, $\mathrm{Mt}=$ mitochondrial, and $\mathrm{Mb}=$ mitochondrial membrane-bound fraction.

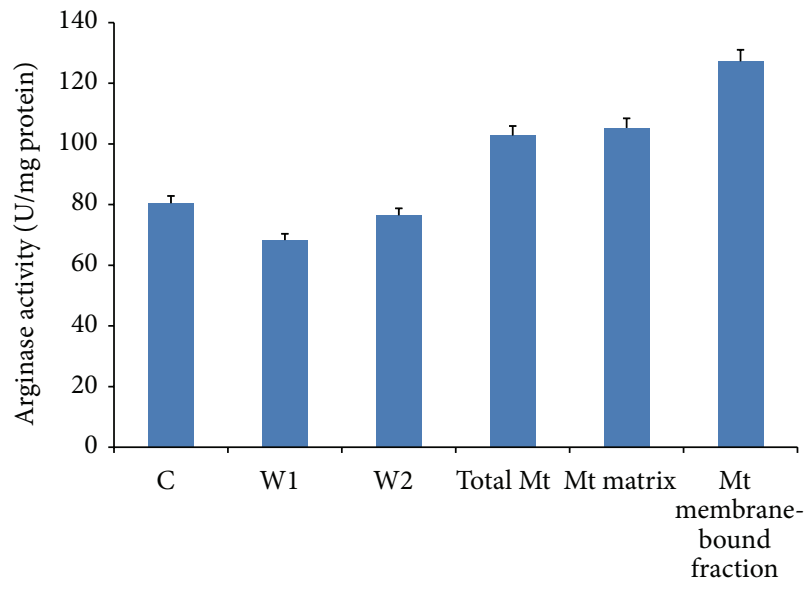

(a)
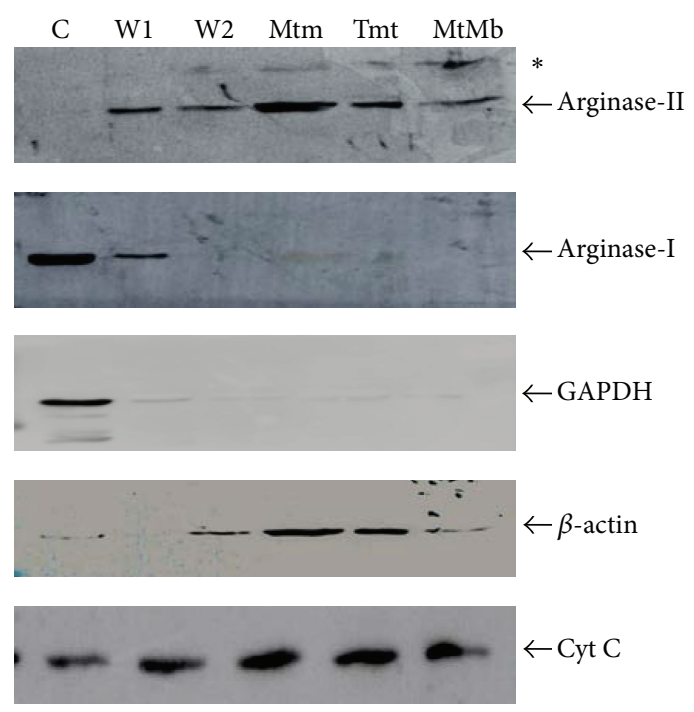

(b)

FIGURE 3: Analysis of arginase isoforms in subcellular fraction of liver of Mus musculus. (a) Histogram represents activity of arginase in different subcellular fractions and (b) shows western blot analysis of cytosolic fraction, total mitochondria, mitochondrial matrix, and mitochondrial outer membrane fractions probed with anti-arginase-II, anti-arginase-I, anti-GAPDH, anti- $\beta$-actin, and anti-cytochrome $\mathrm{C}$ (from top to bottom). The mitochondrial arginase was not observed in cytosolic fraction, whereas it was detected in mitochondrial outer membrane fraction (KCl-treated fraction). The anti-arginase-I did not show cross reactivity with mitochondrial fractions as well as mitochondrial membrane-bound fraction. It clearly indicates that mitochondrial membrane-bound arginase is associated with mitochondrial arginase-II. $\mathrm{C}=$ cytosolic fraction, $\mathrm{W} 1=$ first wash of total mitochondrial fraction with homogenizing medium, W2 = second wash of total mitochondrial fraction with homogenizing medium, $\mathrm{Mtm}=$ mitochondrial matrix fraction (after $\mathrm{KCl}$ wash), Tmt = total mitochondrial fraction (before $\mathrm{KCl}$ treatment), and $\mathrm{MtMb}=$ mitochondrial outer membrane fraction (KCl-washed supernatant). ${ }^{*}$ The variant that is immunoreactive with anti-arginase-II. 


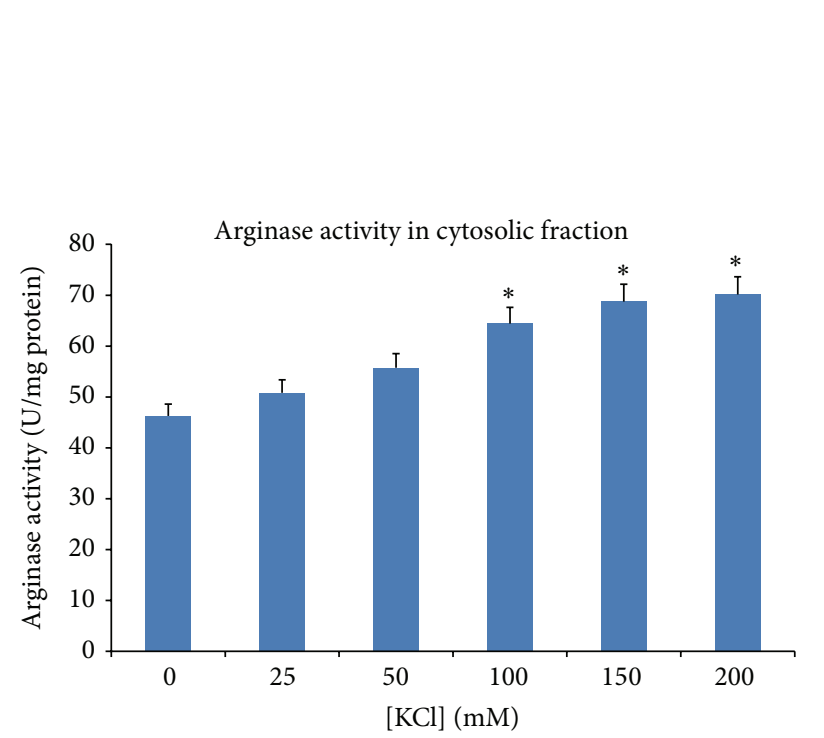

(a)
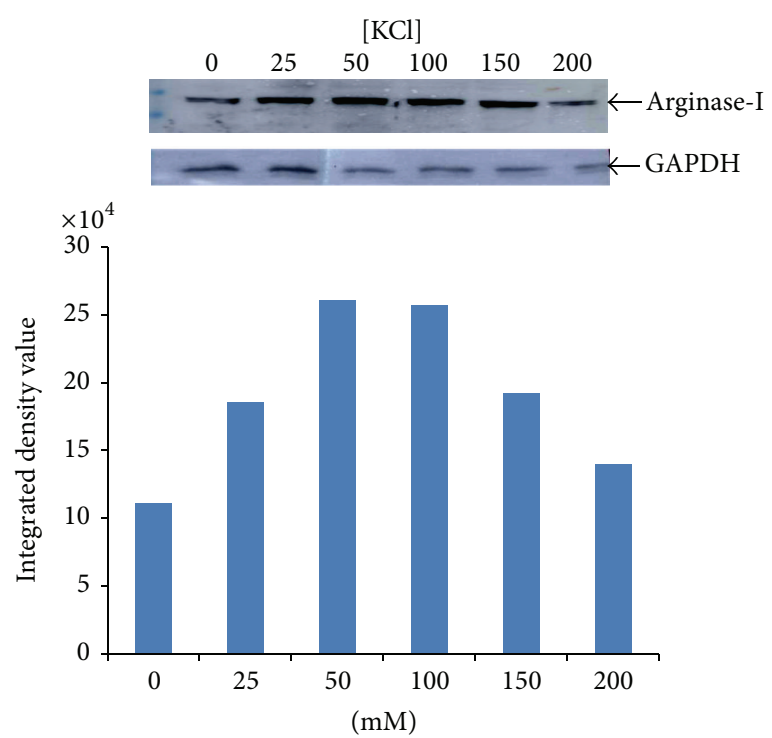

- Arginase-I

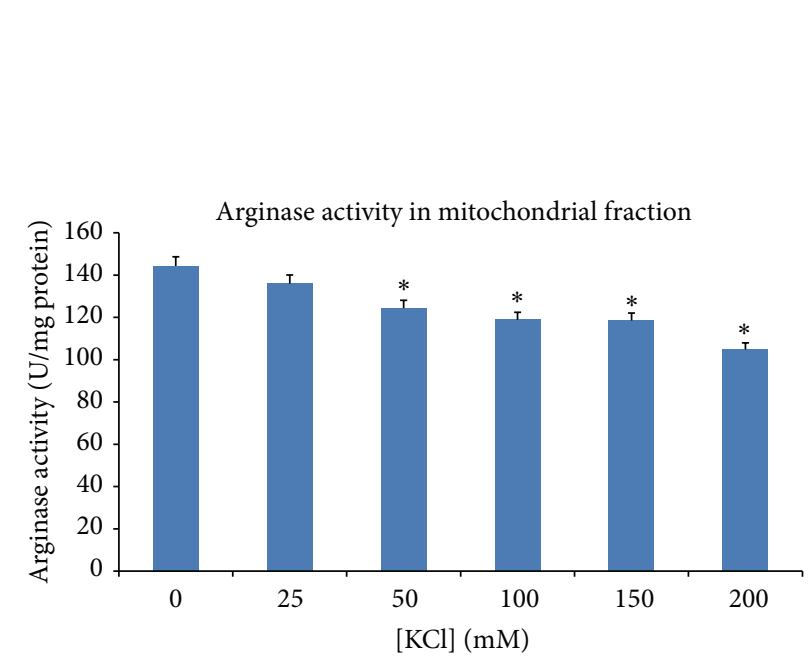

$[\mathrm{KCl}](\mathrm{mM})$ (b)

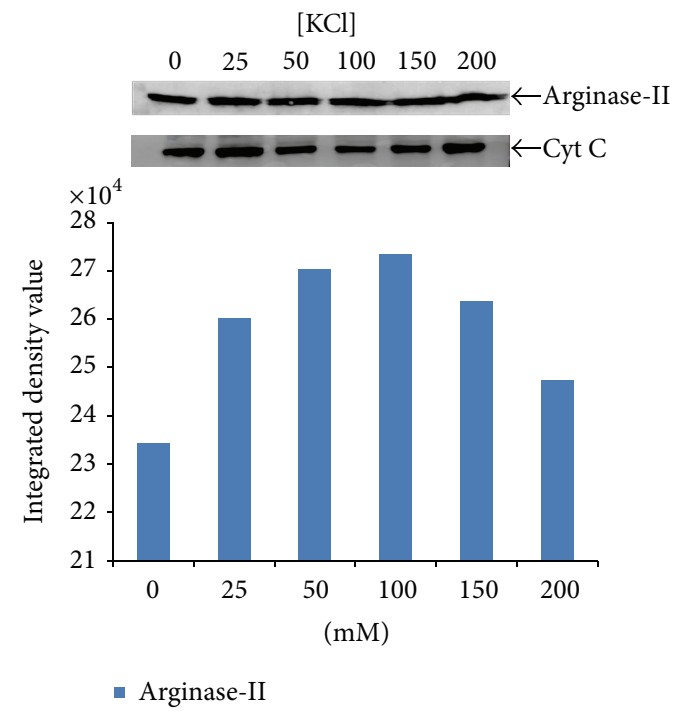

(d)

FIGURE 4: Showing impact of concentration of $\mathrm{KCl}$ in homogenizing medium on the activity of arginase. E1 shows the experimental set 1 described in the text. (a) The activity of arginase in cytosolic fraction. Data is mean of three experiments and histogram represents mean $\pm \mathrm{SD} ;{ }^{*} P<0.05$. (b) The anti-arginase-I cross reacts with the single band of $40 \mathrm{kDa}$ in cytosolic fraction and their activity increases with increasing $\mathrm{KCl}$ concentration in homogenizing medium. Glyceraldehyde 3-phosphate dehydrogenase (GAPDH) is taken as loading control. This result indicates the solubilisation of arginase-I proteins from the membrane of mitochondrial as well as other subcellular organelles. (c) The arginase activity decreases in the mitochondrial fraction with the increasing $\mathrm{KCl}$ concentration. But the percent decrease in the activity of the mitochondrial arginase-II is less than increase in the activity of cytosolic arginase-I; ${ }^{*} P<0.05$. (d) Anti-arginase-II has cross reactivity with the single band of the $40 \mathrm{kDa}$ of mitochondrial fraction. The intensity of this band was decreased with increasing $\mathrm{KCl}$ concentration. Cytochrome C (Cyt C) was taken as loading control.

increasing concentration of $\mathrm{KCl}$ in isolation medium. Simultaneously, the intensity of expected band of anti-arginase-II was decreasing with increasing $\mathrm{KCl}$ concentration in isolation medium from mitochondrial fraction of experiment set 2 (Figures 5(b) and 5(d)). The percent (\%) of arginase activity in mitochondrial membrane-bound fraction was $>50 \%$ of the total mitochondrial arginase activity, which was also found to be increasing progressively with increasing concentration of $\mathrm{KCl}$.

3.3. In Silico Analysis of Arginase and Predicted Alterations in Mitochondrial Targeting and Posttranslational Modifications of Arginase. PCR products ( $1 \mathrm{~kb}, 800 \mathrm{bp}$, and $300 \mathrm{bp})$ of $A R G$ $I$ and $A R G$ II $(1.2 \mathrm{~kb}, 700 \mathrm{bp}$, and $300 \mathrm{bp})$ were observed in 


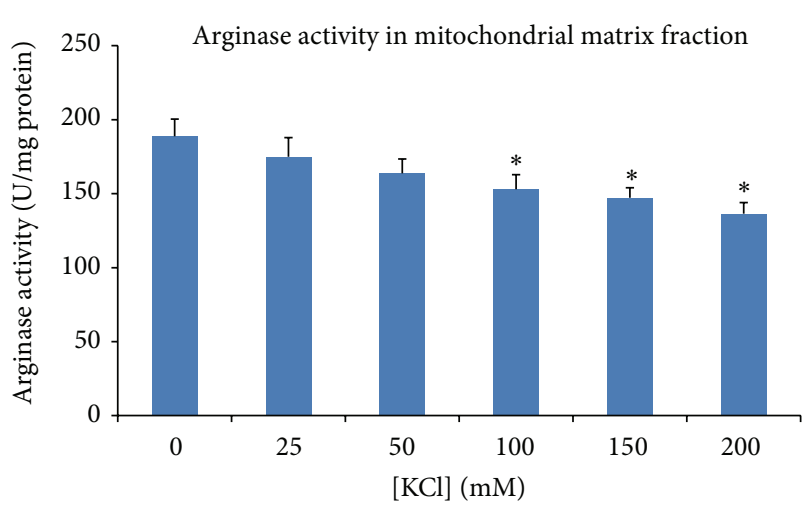

(a)

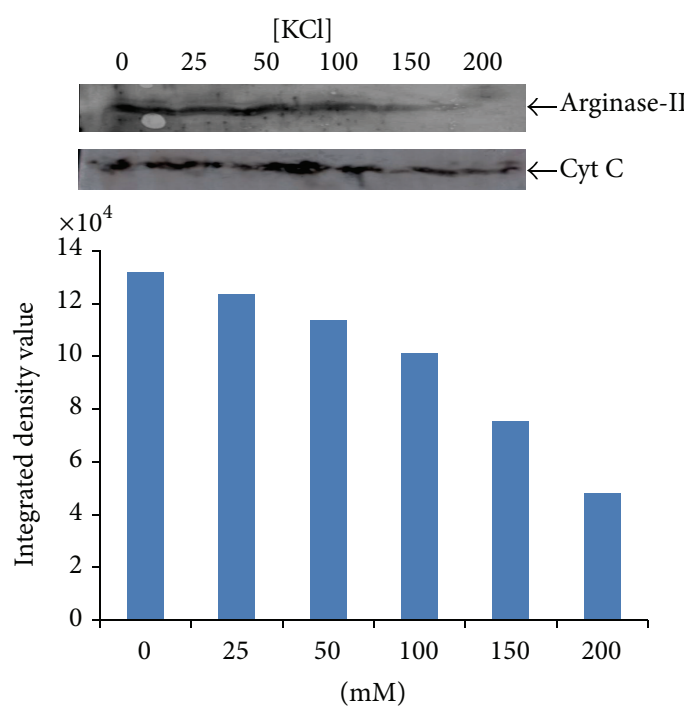

- Arginase-II

(b)
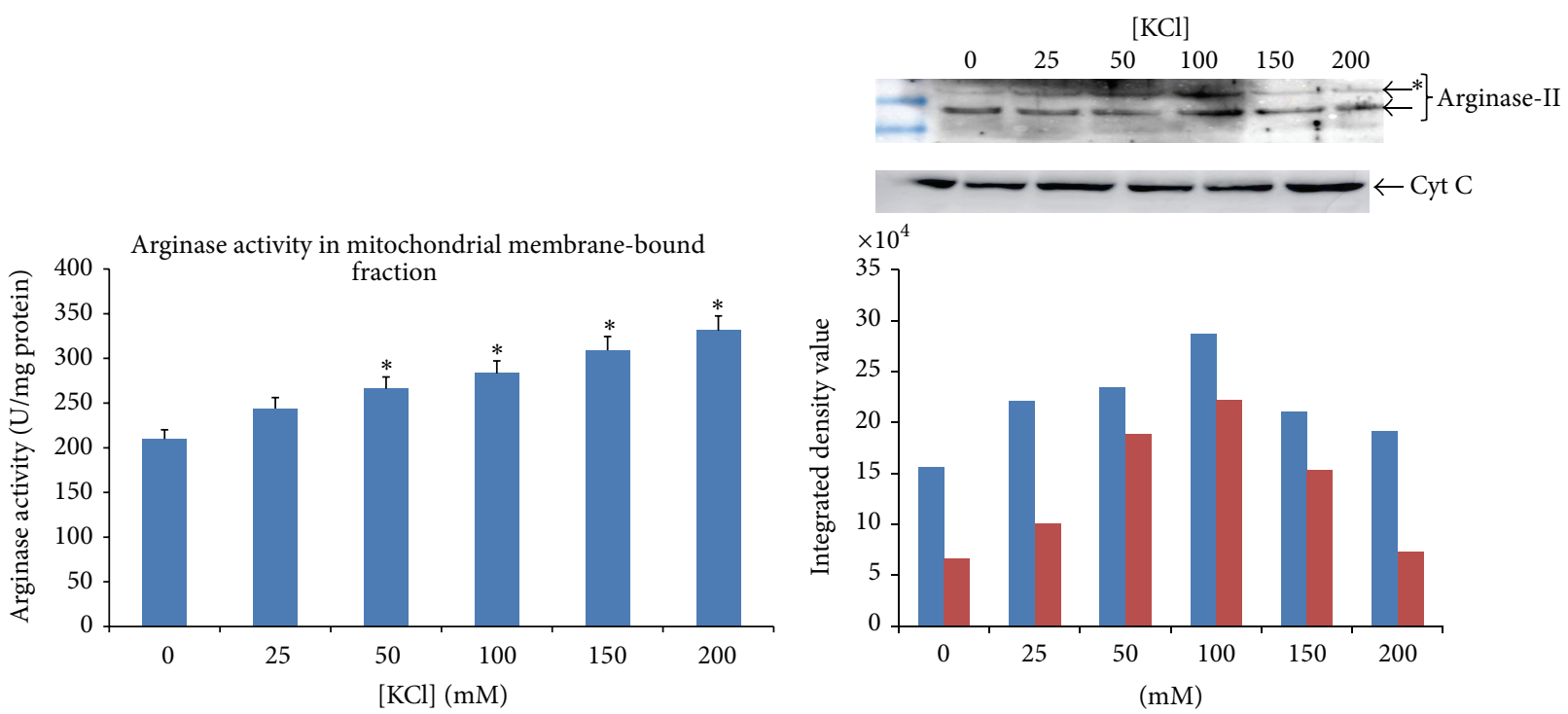

- Arginase-II

- Variant

(d)

FIGURE 5: Data showing the activity of arginase under influence of increasing $\mathrm{KCl}$ concentration on isolated mitochondrial fraction in experiment set 2 in which mitochondria were isolated first and then washed with different concentration of $\mathrm{KCl}$ in homogenizing buffer. (a) Graph shows decreasing activity of arginase with the increasing concentration of $\mathrm{KCl}$ in the homogenizing buffer. Data is mean of three experiments and histogram represents mean $\pm \mathrm{SD} ;{ }^{*} P<0.05$. (b) The mitochondrial matrix fraction probed against anti-arginase-II cross reacts with single band of $40 \mathrm{kDa}$. The intensity of band was decreasing with increasing concentration of $\mathrm{KCl}$. Cytochrome $\mathrm{C}$ (Cyt $\mathrm{C}$ ) was taken as loading control. (c) The increased activity of arginase in membrane-bound fraction was observed with increasing concentration of $\mathrm{KCl}$ in isolation buffer; ${ }^{*} \mathrm{P}<0.05$. (d) The membrane-bound fraction probed against anti-arginase-II has shown band of $40 \mathrm{kDa}$ which is expected for anti-arginase-II along with the band of $50 \mathrm{kDa}$ that shows progressive increase with increasing concentration of $\mathrm{KCl}$; ${ }^{*} \mathrm{The}$ variant that is immunoreactive with anti-arginase-II. Cytochrome C (Cyt C) was taken as loading control.

RT-PCR analysis (Figure 6). Results of western blot analysis and RT-PCR indicated variants of arginase and modulation of arginase-I and arginase-II. The in silico prediction of posttranslational modification of arginase suggests the probability of phosphorylation at serine and threonine residues (http://www.expasy.org/proteomics). Not only phosphorylation but also glycosylation sites were found in arginase-II protein sequence. These modifications may alter the mobility of arginase and association with mitochondrial membrane. The analysis of possible splice variants of 


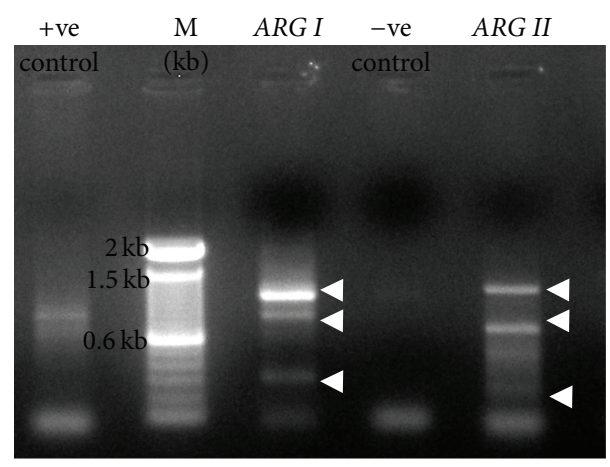

FIGURE 6: RT-PCR analysis of $A R G I$ and $A R G$ II transcripts. Three ARG I (1000 bp, $800 \mathrm{bp}$, and $300 \mathrm{bp}$ ) and three ARG II (1200 bp, $700 \mathrm{bp}$, and $300 \mathrm{bp}$ ) transcripts were indicated by arrow. Bp showed marker, $A R G I=$ arginase-I, and $A R G I I=$ arginase-II. Negative control is reaction mixture without template and positive control primers provided by the manufacturer.

arginase-I and arginase-II (http://www.ensembl.org/; http:// www.genome.ucsc.edu/; http://www.ncbi.nlm.nih.gov/ nucest/) predicts six (ENST00000368087, ENST00000469293, ENST00000356962, ENST00000275196, ENST00000484820, and ENST00000498260) variants of arginase-I in human. However, ARG I seems to have four transcripts variants (ENST00000261783, ENST00000557120, ENST00000556491, and ENST00000557319) in human. The splice variant analysis of Mus musculus ARG II (NM_009705) predicted four high scored splice sites at $261 \mathrm{bp}, 388 \mathrm{bp}$, $407 \mathrm{bp}$, and $455 \mathrm{bp}$ with scores of $8.571,7.898,7.542$, and 9.505, respectively, by ASSP server. For ARG I (NM_007482), three high scored splice sites at $240 \mathrm{bp}, 346 \mathrm{bp}$, and $378 \mathrm{bp}$ with scores of 9.402, 7.223, and 7.612, respectively, were predicted by ASSP. Similarly, EST sequences of ARG II were observed in the range from $300 \mathrm{bp}$ to $1024 \mathrm{bp}$. The virtual translation products of $1024 \mathrm{bp}$ (DV039756) encode 197aa, 965 bp (DV074609), and 308aa. Observations provide us insight into the fact that the PCR products of ARG I $(1 \mathrm{~kb}$, $800 \mathrm{bp}$, and $300 \mathrm{bp}$ ) and arginase-II $(1.2 \mathrm{~kb}, 700 \mathrm{bp}$, and $300 \mathrm{bp}$ ) could be the splice variants of arginase.

The physicochemical analysis and posttranslational modification of arginase-I and arginase-II were summarised in Table 1. Several posttranslational sites such as phosphorylation, glycosylation, N-O glycosylation, and acetylation sites were predicted in arginase-I and arginase-II. Analysis through MitoProt-II and iPSORT shows signal peptide "MFLRSSASRLLHGQIPCVLTRSVHSVAIVG" in arginaseII but not in arginase-I that is essential for the targeting of arginase-II to mitochondria. The analysis of virtual mutagenesis of the predicted targeting sequence of arginase-II revealed that amino acid residues from $\mathrm{N}$ terminus "MFL." do not affect targeting. However, arginine $(R)$, serine $(S)$, and stretch of seven residues "SRLLHGQ" out of "MFLRSSASRLLHGQIPCVLTRSVHSVAIVG" are very critical. The cleavage site between "MFLRSSASRLL." and "HGQIPCVLTRSVHSVAIVG" was also identified. Such alterations in mitochondrial targeting sequences of
arginase-II may alter transport and localization of arginaseII either on inner or on outer membrane of mitochondria (Figure 8).

3.4. Ultrastructural Changes Were Observed after KCl Treatment to Mitochondria. The analysis by TEM indicates $\mathrm{KCl}-$ dependent swelling of mitochondria and disruption of the outer mitochondrial membrane (Figure 7(a)). The deformities in the cristae of KCl-treated mitochondria (Figure 7(b)) were evident as compared to control.

\section{Discussion and Conclusion}

The arginase-I facilitates removal of nitrogenous wastes and also serves as competitor substrate of the nitric oxide synthetase regulating nitric oxide production. However, mitochondrial arginase-II necessitates production of ornithine, proline, and glutamate required for synthesis of proteins and collagen. Since both isoforms get localised in different subcellular locations, they can efficiently utilize different pool of substrate. They help in efficient channelling of arginine and ornithine from mitochondria to cytosol and vice versa. Since transporters of arginine and ornithine have been found localised on the mitochondrial membrane along with $\mathrm{K}^{+}$ channels, the $\mathrm{KCl}$ may induce alterations in opening of mitochondrial permeability transition pores. Observations suggest $\mathrm{KCl}$-dependent release of arginase and also effect of $\mathrm{KCl}$ on mitochondrial integrity. Results provided insight about proteins released from mitochondria because the mitochondria were isolated first without $\mathrm{KCl}$ in homogenizing medium; after that the mitochondrial pellet was washed with $0-200 \mathrm{mM} \mathrm{KCl}$ in homogenizing medium (Figure 5). Arginase activity in $\mathrm{KCl}$-washed fractions suggested that the mitochondrial membrane-bound arginase could be the mitochondrial arginase released under influence of $\mathrm{KCl}$ (Figure 8). This specific polypeptide could be a variant of arginase-II (Figure 3(b)) and is likely to be presumed mitochondrial membrane-bound fraction. Since the second wash (W2) removed all the disrupted mitochondrial content, the band detected in the membrane-bound fraction was released under influence of the $\mathrm{KCl}$. Observations (Figures 3-5) also confirmed that the increasing mitochondrial arginase activity in membrane-bound fraction leaked as the membrane-bound fraction from the mitochondrial matrix. Findings also indicate that the arginase-I does not remain associated with membrane of mitochondria. If it would have been so, the levels of arginase-I would have been higher, not arginase-II. Higher level of arginase activity under influence of $\mathrm{KCl}$ seems to be due to the leakage of mitochondrial arginase-II because activity of arginase-II got decreased and intensity of arginase-II reactive band increased. The decrease in the mitochondrial matrix arginase-II confirms the leakage of mitochondrial enzyme at higher concentration of $\mathrm{KCl}$. It seems that mitochondrial membrane-bound arginase and arginase-II contain similar epitope but may differ in charge or get unique molecular signature due to posttranslational modifications. Earlier, different isoforms of arginase have also been reported $[13,14,16,17]$ but our results suggest that the mitochondrial membrane-bound arginase could be 


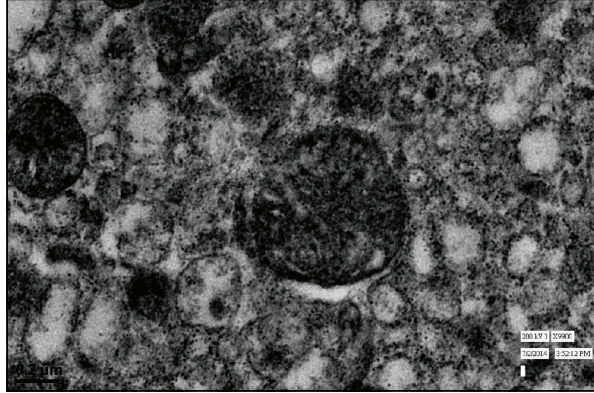

(a)

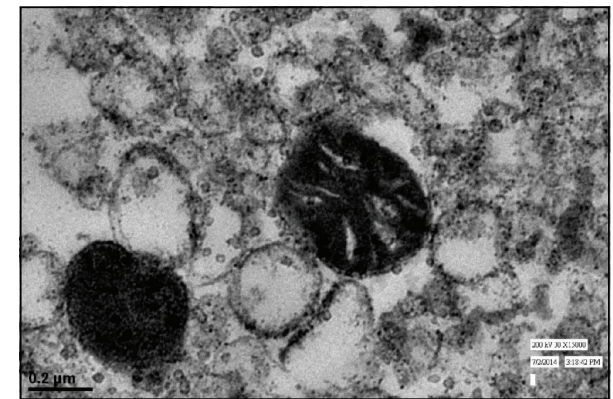

(b)

FIGURE 7: Transmission electron microscopic analysis of mitochondrial surface before and after the treatment of $\mathrm{KCl}$ showed mitochondrial swelling, altered morphology of cristae, and disruption in outer mitochondrial membrane after $\mathrm{KCl}$ treatment. (a) Ultrastructure of control mitochondria and (b) ultrastructure of $\mathrm{KCl}$-treated mitochondria.

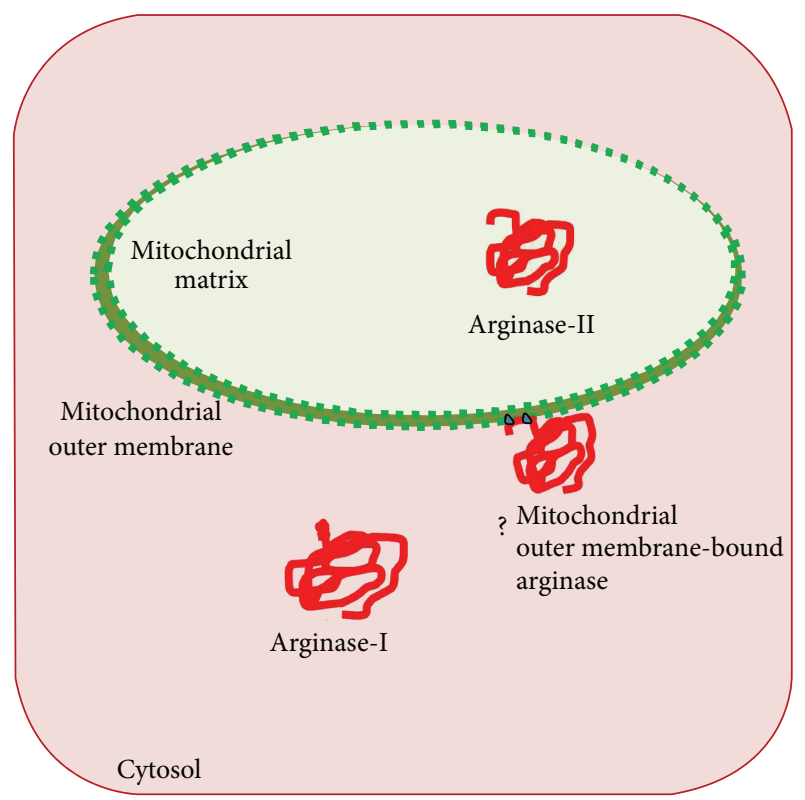

Figure 8: Illustration of presumed state of mitochondrial membrane-bound arginase. The question mark indicates the presence of mitochondrial membrane-bound arginase that may have been mistargeted due to posttranslational modification or alterations in the signal peptide.

mitochondrial arginase, not the cytosolic arginase (Figure 3). A similar observation [32] also reveals that arginase associated with plasma membrane did not show cross reactivity with the liver cytosolic arginase.

The in silico analysis of arginase-I and arginase-II with MitoProt (http://ihg.gsf.de/ihg/mitoprot.html) [33] and iPSORT (http://ipsort.hgc.jp/predict.cgi) [34] also suggested that critical amino acid residues arginine (R), serine (S), and stretch of seven residues "SRLLHGQ" in the signal peptide of arginase-II seem critical for targeting. Since cleavage between "MFLRSSASRLL." and "HGQIPCVLTRSVHSVAIVG" may lead to mistargeting of arginase-II to other organelles, it is likely that posttranslational modifications, particularly phosphorylation to serine $(\mathrm{S})$, proline $(\mathrm{P})$, and threonine
(T) residues, may alter targeting to mitochondrial matrix and remain associated with the membrane (Table 1). Such phosphorylated arginase-II might have altered mobility (Figure 5(d)) and is detected as a variant of arginase-II associated with mitochondrial outer membrane (Figure 8). The in silico prediction of posttranslational modification also suggests the probability of phosphorylation at serine and threonine residues (http://www.expasy.org/proteomics). Not only phosphorylation but also glycosylation sites were predicted in arginase-II protein sequence that may alter the mobility of arginase and association with mitochondrial membrane. The important spliced variants and/or ESTs of $A R G I$ and $A R G$ II ranging from $132 \mathrm{bp}$ (AW113714) to 1,166 bp (BI649622) also support the idea of possible splice variant. The virtual translation of matching UniGene sequences DV039756 and DV074609 provides insight that the PCR products (Figure 6) of $A R G I(1 \mathrm{~kb}, 800 \mathrm{bp}$, and $300 \mathrm{bp})$ and $A R G I I(1.2 \mathrm{~kb}, 700 \mathrm{bp}$, and $300 \mathrm{bp}$ ) could be the splice variants of arginase.

Thus, mitochondrial-membrane bound arginase seems similar to arginase-II, a novel variant, or could be arginaseII having posttranslational modifications which is localized to mitochondria for a specialized function.

\section{Competing Interests}

The authors declare that they have no competing interests.

\section{Acknowledgments}

Mishra Suman acknowledges the Research Fellowship in Science for Meritorious Students, UGC, New Delhi. The authors acknowledge financial support from BHU, UGCCAS, UGC-UPE, and BHU-DBT-ISLS. They also appreciate AIIMS, New Delhi, for extending TEM facility for this study.

\section{References}

[1] A. Kossel and H. D. Dakin, "Über die arginase," Hoppe-Seyler's Zeitschrift für Physiologische Chemie, vol. 41, no. 4, pp. 321-331, 1904.

[2] J. Kitayama, S. Emoto, H. Yamaguchi et al., "CD90(+)CD45(-) intraperitoneal mesothelial-like cells inhibit $\mathrm{T}$ cell activation by 
production of arginase I," Cellular Immunology, vol. 288, no. 1, pp. 8-14, 2014.

[3] M. Mori and T. Gotoh, "Arginine metabolic enzymes, nitric oxide and infection," Journal of Nutrition, vol. 134, no. 10, pp. 2820S-2825S, 2004.

[4] N. Goncharova and E. M. Muntian, "The role of bivalent cations in the binding of hexokinase II isoenzyme to mitochondrial membranes," Biokhimiia, vol. 51, no. 5, pp. 801-807, 1986.

[5] D. Brdiczka, "Function of the outer mitochondrial compartment in regulation of energy metabolism," Biochimica et Biophysica Acta (BBA)-Bioenergetics, vol. 1187, no. 2, pp. 264-269, 1994.

[6] W. Durante, F. K. Johnson, and R. A. Johnson, "Arginase: a critical regulator of nitric oxide synthesis and vascular function," Clinical and Experimental Pharmacology and Physiology, vol. 34, no. 9, pp. 906-911, 2007.

[7] M. Mori, "Regulation of nitric oxide synthesis and apoptosis by arginase and arginine recycling," Journal of Nutrition, vol. 137, no. 6, pp. 1616S-1620S, 2007.

[8] O. Savu, L. Iosif, O. M. Bradescu, C. Serafinceanu, R. Papacocea, and I. Stoian, "L-arginine catabolism is driven mainly towards nitric oxide synthesis in the erythrocytes of patients with type 2 diabetes at first clinical onset," Annals of Clinical Biochemistry, vol. 52, no. 1, pp. 135-143, 2015.

[9] A. Chrzanowska, W. Graboń, M. Mielczarek-Puta, and A. Barańczyk-Kuźma, "Significance of arginase determination in body fluids of patients with hepatocellular carcinoma and liver cirrhosis before and after surgical treatment," Clinical Biochemistry, vol. 47, no. 12, pp. 1056-1059, 2014.

[10] P. Y. Chan and E. A. Cossins, "Arginine metabolism in Saccharomyces cerevisiae. Some general properties of yeast arginase," Plant and Cell Physiology, vol. 14, no. 4, pp. 641-651, 1973.

[11] S. Ljubisavljevic, I. Stojanovic, R. Pavlovic, and D. Pavlovic, “The importance of nitric oxide and arginase in the pathogenesis of acute neuroinflammation: are those contra players with the same direction?" Neurotoxicity Research, vol. 26, no. 4, pp. 392399, 2014.

[12] R. B. Caldwell, H. A. Toque, S. P. Narayanan, and R. W. Caldwell, "Arginase: an old enzyme with new tricks," Trends in Pharmacological Sciences, vol. 36, no. 6, pp. 395-405, 2015.

[13] I. Gasiorowska, Z. Porembska, J. Jachimowicz, and I. Mochnacka, "Isoenzymes of arginase in rat tissues," Acta Biochimica Polonica, vol. 17, no. 1, pp. 19-30, 1970.

[14] G. A. Kaysen and H. J. Strecker, "Purification and properties of arginase of rat kidney," Biochemical Journal, vol. 133, no. 4, pp. 779-788, 1973.

[15] C. A. Casey and P. M. Anderson, "Subcellular location of glutamine synthetase and urea cycle enzymes in liver of spiny dogfish (Squalus acanthias)," The Journal of Biological Chemistry, vol. 257, no. 14, pp. 8449-8453, 1982.

[16] C. P. Jenkinson, W. W. Grody, and S. D. Cederbaum, "Comparative properties of arginases," Comparative Biochemistry and Physiology Part B: Biochemistry and Molecular Biology, vol. 114, no. 1, pp. 107-132, 1996.

[17] R. Tarrab, J. Rodriguez, C. Huitron, R. Palacios, and G. Soberón, "Molecular forms of rat liver arginase. Isolation and characterization," European Journal of Biochemistry, vol. 49, no. 2, pp. 457468,1974 .

[18] A. Herzfeld and S. M. Raper, "The heterogeneity of arginases in rat tissues," Biochemical Journal, vol. 153, no. 2, pp. 469-478, 1976.
[19] G. Venkatakrishnan and S. R. R. Reddy, "Arginase isoforms in frog and lizard tissues," Indian Journal of Biochemistry and Biophysics, vol. 47, no. 1, pp. 13-19, 2010.

[20] Z. Porembska and E. Zamecka, "Immunological properties of rat arginases," Acta Biochimica Polonica, vol. 31, no. 2, pp. 223227, 1984.

[21] S. Traniello, R. Barsacchi, E. Magri, and E. Grazi, "Molecular characteristics of chicken kidney arginase," Biochemical Journal, vol. 145, no. 2, pp. 153-157, 1975.

[22] I. Skrzypek-Osiecka, I. Rahden-Staroń, and Z. Porembska, "Sub-cellular localization of arginase in rat liver," Acta Biochimica Polonica, vol. 27, no. 3-4, pp. 203-212, 1980.

[23] C.-W. Cheung and L. Raijman, "Arginine, mitochondrial arginase, and the control of carbamyl phosphate synthesis," Archives of Biochemistry and Biophysics, vol. 209, no. 2, pp. 643649, 1981.

[24] I. Nissim, B. Luhovyy, O. Horyn, Y. Daikhin, I. Nissim, and M. Yudkoff, "The role of mitochondrially bound arginase in the regulation of urea synthesis: studies with $\left[\mathrm{U}^{15} \mathrm{~N}_{4}\right]$ arginine isolated mitochondria and perfused rat liver," The Journal of Biological Chemistry, vol. 280, no. 18, pp. 17715-17724, 2005.

[25] S. Srivastava and B. K. Ratha, "Unique hepatic cytosolic arginase evolved independently in ureogenic freshwater air-breathing teleost, heteropneustes fossilis," PLoS ONE, vol. 8, no. 6, Article ID e66057, 2013.

[26] R. D. Glass and W. E. Knox, "Arginase isozymes of rat mammary gland, liver, and other tissues," The Journal of Biological Chemistry, vol. 248, pp. 5785-5789, 1973.

[27] A. Devin, B. Guérin, and M. Rigoulet, "Response of isolated rat liver mitochondria to variation of external osmolarity in $\mathrm{KCl}$ medium: regulation of matrix volume and oxidative phosphorylation," Journal of Bioenergetics and Biomembranes, vol. 29, no. 6, pp. 579-590, 1997.

[28] L. Michea, C. Combs, P. Andrews, N. Dmitrieva, and M. B. Burg, "Mitochondrial dysfunction is an early event in high$\mathrm{NaCl}$-induced apoptosis of mIMCD3 cells," American Journal of Physiology-Renal Physiology, vol. 282, no. 6, pp. F981-F990, 2002.

[29] H. A. Ells, "A colorimetric method for the assay of soluble succinic dehydrogenase and pyridinenucleotide-linked dehydrogenases," Archives of Biochemistry and Biophysics, vol. 85, no. 2, pp. 561-562, 1959.

[30] M. M. Bradford, "A rapid and sensitive method for the quantitation of microgram quantities of protein utilizing the principle of protein-dye binding," Analytical Biochemistry, vol. 72, no. 1-2, pp. 248-254, 1976.

[31] G. W. Brown Jr. and P. P. Cohen, "Comparative biochemistry of urea synthesis. I. Methods for the quantitative assay of urea cycle enzymes in liver," The Journal of Biological Chemistry, vol. 234, no. 7, pp. 1769-1774, 1959.

[32] J. M. Fuentes, M. L. Campo, and G. Soler, "Physico-chemical properties of hepatocyte plasma-membrane-bound arginase," Archives Internationales de Physiologie, de Biochimie et de Biophysique, vol. 99, no. 6, pp. 413-417, 1991.

[33] M. G. Claros and P. Vincens, "Computational method to predict mitochondrially imported proteins and their targeting sequences," European Journal of Biochemistry, vol. 241, no. 3, pp. 779-786, 1996.

[34] H. Bannai, Y. Tamada, O. Maruyama, K. Nakai, and S. Miyano, "Extensive feature detection of N-terminal protein sorting signals," Bioinformatics, vol. 18, no. 2, pp. 298-305, 2002. 

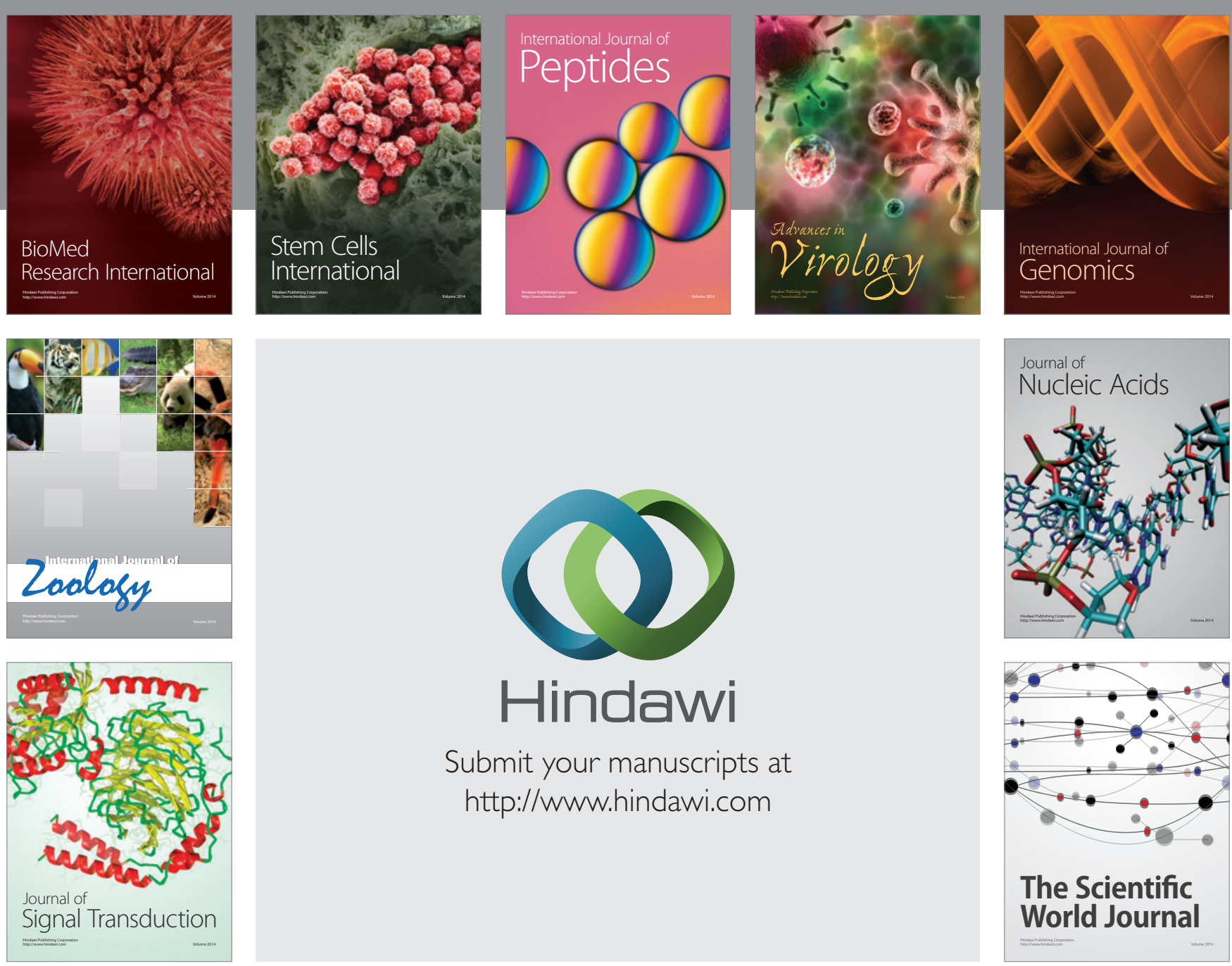

Submit your manuscripts at

http://www.hindawi.com
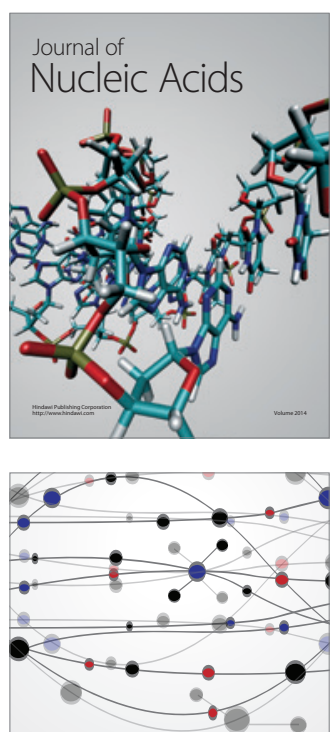

The Scientific World Journal
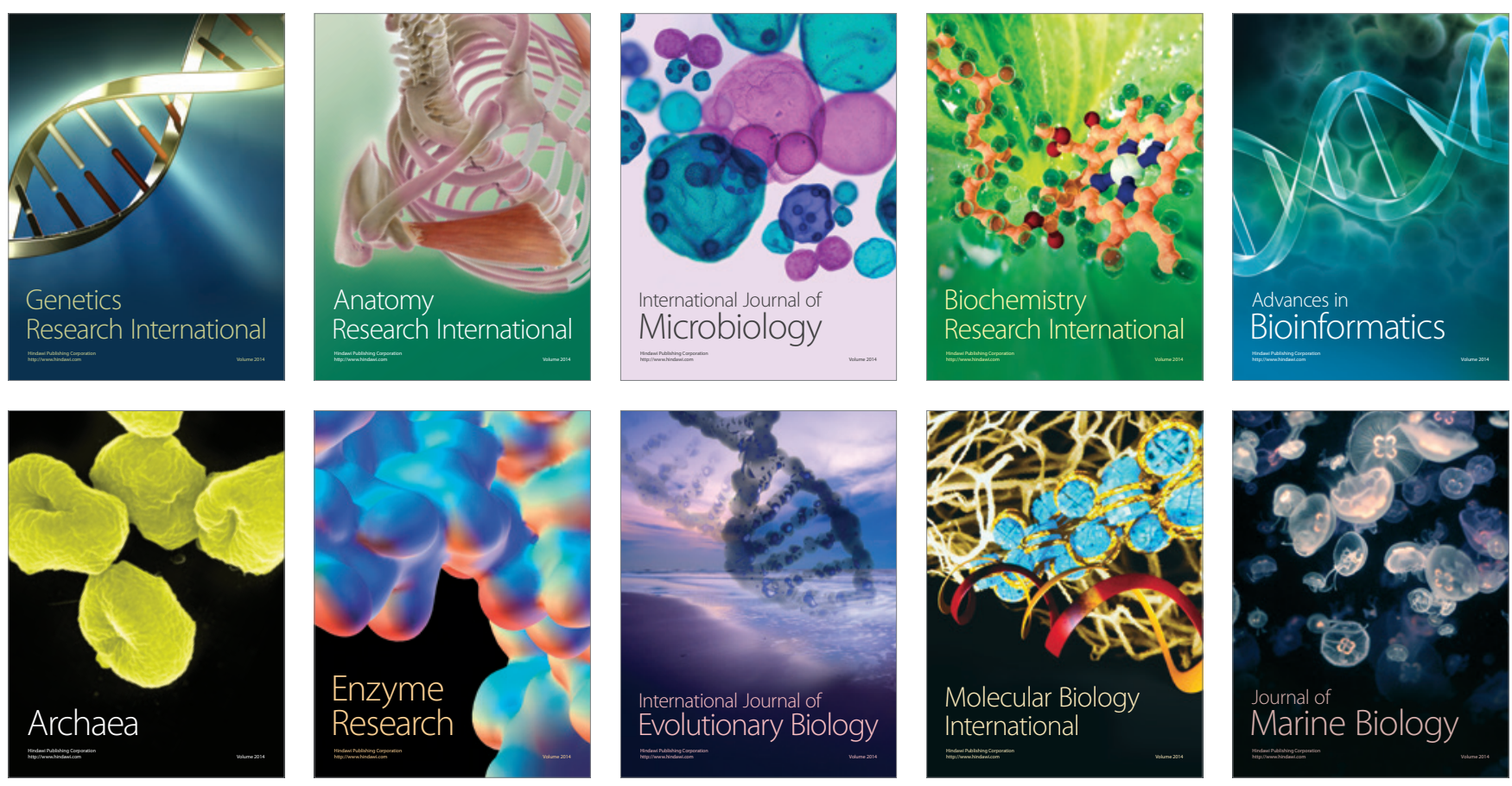